\title{
Neuroprotective effects of erythropoietin against oxidant injury following brain irradiation: an experimental study
}

Gamze Ugurluer ${ }^{1}$, Aysegul Cebi ${ }^{2}$, Handan Mert ${ }^{3}$, Nihat Mert ${ }^{3}$, Meltem Serin ${ }^{1}$, Haldun Sukru Erkal ${ }^{4}$

\author{
'Department of Radiation Oncology, Faculty of Medicine, Acibadem University, \\ Acibadem Adana Hospital, Adana, Turkey \\ ${ }^{2}$ Department of Food Engineering, Faculty of Engineering, Giresun University, Giresun, \\ Turkey \\ ${ }^{3}$ Department of Biochemistry, Faculty of Veterinary, Yuzuncu Yil University, Van, Turkey \\ ${ }^{4}$ Department of Radiation Oncology, Faculty of Medicine, Sakarya University, Sakarya, \\ Turkey
}

Submitted: 13 November 2014

Accepted: 16 November 2014

Arch Med Sci 2016; 12, 6: 1348-1353

DOI: $10.5114 /$ aoms.2016.58622

Copyright $\odot 2016$ Termedia \& Banach

\section{Abstract}

Introduction: Radiation therapy (RT) is a major treatment modality, and the central nervous system is a dose-limiting organ in clinical RT. This experimental study aims to present the evaluation of the neuroprotective effects of erythropoietin (EPO) against oxidant injury following brain irradiation in rats.

Material and methods: Forty Wistar rats were randomly assigned to four groups ( $n=10$ each). In group 1 the rats received no EPO and underwent sham RT. The rats in groups 2 and 3 received EPO. In group 2 rats underwent sham RT, while in group 3 rats received RT. The rats in group 4 received no EPO and underwent RT. Rats were irradiated using a Cobalt- 60 teletherapy machine using a single fraction of 20 Gy covering the whole brain. Cervical dislocation euthanasia was performed. The nitrite and malondialdehyde (MDA) levels and the superoxide dismutase (SOD) and glutathione peroxidase (GSHPX) activities were evaluated in dissected brain tissues.

Results: The nitrite and MDA levels were higher in the RT group $(2.10 \pm 0.62$ ppm, $26.02 \pm 2.16 \mathrm{nmol} / \mathrm{ml} ; p<0.05)$ and lower in the EPO + RT group (1.45 $\pm 0.12 \mathrm{ppm}, 25.49 \pm 1.90 \mathrm{nmol} / \mathrm{ml} ; p<0.05)$. The SOD and GSHPX activity was higher in the EPO + RT group $(2.62 \pm 0.49 \mathrm{U} / \mathrm{mg}, 1.75 \pm 0.25 \mathrm{U} / \mathrm{mg}, p<0.05)$.

Conclusions: This study supports the probable neuroprotective effects of EPO against oxidant injury following brain irradiation in a rat model, presumably through decreasing free radical production and increasing expression of antioxidant enzymes.

Key words: brain, irradiation, oxidant injury, neuroprotective effects, erythropoietin.

\section{Introduction}

The central nervous system (CNS) is a dose-limiting organ in clinical radiation therapy (RT) [1]. Irradiation causes injury to the cellular DNA content, and this is most commonly a result of the reactive oxygen/nitrogen oxide species (ROS/RNOS) [2,3]. The ROS/RNOS include oxygen ions, free radicals and both inorganic and organic peroxides (i.e., the su-

\author{
Corresponding author: \\ Gamze Ugurluer MD \\ Department \\ of Radiation Oncology \\ Faculty of Medicine \\ Acibadem University \\ Acibadem Adana Hospital \\ Cumhuriyet Caddesi No 66 \\ 01130 Adana, Turkey \\ Phone: +90 5322509172 \\ Fax: +90 3224554477 \\ E-mail: gamze.ugurluer@ \\ acibadem.edu.tr
}


peroxide radical $\left(\mathrm{O}_{2}^{-}\right)$, the hydroxyl radical $(\mathrm{OH})$, nitric oxide (NO) and hydrogen peroxide $\left(\mathrm{H}_{2} \mathrm{O}_{2}\right)$ ), which are highly reactive due to the presence of unpaired electrons in their shells [4]. Nitric oxide is a free radical induced by irradiation and has cytotoxic effects [5]. Due to the very short half-life of $\mathrm{NO}$ in aqueous solutions, it is generally measured indirectly via its metabolites (nitrate and nitrite), collectively referred to as reactive nitrogen intermediates. The hydroxyl radical reacts with extremely high rate constants with almost every type of molecule found in living cells: sugars, amino acids, phospholipids, DNA bases and organic acids [6]. Mechanisms have evolved to restrict and control such processes, partly by compartmentation, and partly by antioxidant defenses such as chain-breaking antioxidant compounds capable of forming stable free radicals and the evolution of enzyme systems (e.g. superoxide dismutase (SOD), catalase (CAT), and glutathione peroxidase (GSH$\mathrm{PX})$ ) that diminish the intracellular concentration of the ROS [7]. Malondialdehyde (MDA), a naturally occurring product of lipid peroxidation and prostaglandin biosynthesis, is one of the most frequently used indicators of lipid peroxidation [8]. However, during times of environmental stress, the ROS levels can increase dramatically, overwhelming antioxidant systems and resulting in significant damage to cell structures [3]. This scenario is referred to as oxidative stress. Under normal conditions, ROS/ RNOS generation is approximately in balance with the cell's antioxidant defenses (antioxidants/antioxidant enzymes). Any imbalance between ROS/ RNOS generation and destruction in favor of ROS/ RNOS generation can create oxidative stress. The brain has relatively modest antioxidant systems [9]. Cancer patients, with many ongoing improvements in health care, survive long enough for the adverse radiation effects to become clinically significant and affect quality of life. Therefore, the development of any neuroprotective treatment that reduces radiation-related side effects is clearly desirable.

Erythropoietin (EPO) is a cytokine produced in the kidney that is predominantly involved in the proliferation, maturation and differentiation of the precursors of the erythroid cells. Human recombinant EPO has been used for over a decade to treat anemia in uremic as well as cancer patients [1]. Erythropoietin and its receptor, EpoR, have been shown to be expressed in rodent and human brains, in cultured neurons, astrocytes, oligodendrocytes, microglia, and endothelial cells, which have led to studies investigating the additional biological roles of EPO [10]. Erythropoietin and EpoR in the CNS and the upregulation of EPO by hypoxia/ischemia in vitro and in vivo suggest that this cytokine is an important mediator of the response of the brain to injury [11]. The sug- gested neuroprotective effects of EPO include antagonism against the deleterious action of glutamate, increase in the expression of antioxidant enzymes, decrease in free-radical production, augmentation in the release of neurotransmitters, increased pro-angiogenic activity and induction of neuroglobin (an intracerebral $\mathrm{O}_{2}$ transporter) [12]. Despite its large molecular size (37 kD) and its highly glycosylated and negatively charged structure, systemic EPO crosses the blood-brain barrier in a dose-dependent manner to peak in the brain at 6 to $10 \mathrm{~h}$ [13]. Based on previously published animal studies, EPO is neuroprotective at 5000 IU/ $\mathrm{kg}$ [13]. Although the neuroprotective effects of EPO have been well documented in experimental models of brain and spinal cord injury, this experimental study aims to present the evaluation of the neuroprotective effects of EPO against oxidant injury following brain irradiation.

\section{Material and methods}

\section{Experimental design}

The experimental study procedures followed the "Guide for the Care and Use of Laboratory Animals (Canadian Council on Animal Care)". It was done at the "Inonu University Experimental Studies Laboratory". The protocol approval was obtained from "Inonu University Animal Ethics Committee". Forty female Wistar rats were randomly assigned to four groups ( $n=10$ each). They were four months old and weighed between 160 and $180 \mathrm{~g}$. The rats were individually housed in rooms with controlled temperature and light. Standard chow and tap water were freely available. Group 1 was the control group and the rats did not receive EPO and underwent sham RT. Erythropoietin was given to the rats in groups 2 and 3 . In group 2 rats underwent sham RT (EPO group), while in group 3 rats received RT (EPO + RT group). Group 4 was the RT group and the rats did not receive EPO and received RT. Erythropoietin (NeoRecormon, Roche, Mannheim, Germany) was prepared with dilution in $0.9 \%$ sodium chloride. It was administered (5000 IU/kg body weight) by intraperitoneal injection $8 \mathrm{~h}$ prior to RT or sham RT. For the rats in control and RT groups, the same volume $(0.9 \%)$ of sodium chloride was administered by intraperitoneal injection.

\section{Irradiation and euthanasia}

The rats were irradiated in a prone position using a Cobalt-60 teletherapy machine. The fraction dose was 20 Gy and a single anterior portal with a $0.5 \mathrm{~cm}$ bolus covered the whole brain. The dose was defined at a depth of $2.5 \mathrm{~cm}$. Ketamine (Ketalar, Pfizer, Istanbul, Turkey, $80 \mathrm{mg} / \mathrm{kg}$ body weight) and xylazine (Rompun, Bayer, Istanbul, Turkey, 
$5 \mathrm{mg} / \mathrm{kg}$ body weight) anesthesia was used and administered by intraperitoneal injection before irradiation. A customized restrainer was used for immobilization. Sham RT was also given on a Cobalt-60 teletherapy machine using the same duration. The rats were observed until they were completely recovered from the anesthesia. The cervical dislocation euthanasia was performed at $2 \mathrm{~h}$ following RT or sham RT.

\section{Biochemical evaluation}

The brains were dissected and frozen in crushed dry ice. The tissues were stored at $-35^{\circ} \mathrm{C}$ until biochemical evaluation. Cold phosphate buffered saline (PBS) solution $(50 \mathrm{mM}, \mathrm{pH} 7.4$, with a ratio of $1: 5)$ was used for perfusion to prevent blood contamination before tissue homogenization. A homogenizer (Yellow line DI-25 Basic, IKA Werke $\mathrm{GmbH}$, Staufen, Germany) was used and the temperature was kept at $+4^{\circ} \mathrm{C}$ during the process.

Total nitrate and nitrite concentrations were measured as the stable end product, nitrite, according to Stahr's modification method and determined spectrophotometrically at $520 \mathrm{~nm}$ [14]. For measurement of MDA level, homogenate and phosphoric acid were mixed and then heated for $45 \mathrm{~min}$ after the addition of thiobarbituric acid [15]. The absorbance of the complex was measured on a spectrophotometer (Boeco S-22 UV/ Vis, Hamburg, Germany).

The homogenate was centrifuged for $15 \mathrm{~min}$ using a centrifuge (Heraeus Sepatech Minifuge RF, Heraeus Instruments $\mathrm{GmbH}$, Hanau, Germany). The assay for SOD activity was made according to the method of Suttle using a Ransel kit (Randox Labs., Crumlin, UK) [16]. This method is based on the formation of red formazan from the reaction of 2-(4-iodophenyl)-3-(4-nitrophenol)-5-phenyltetrazolium chloride and superoxide radical (produced in the incubation medium from xanthine oxidase reaction), which is assayed in a spectrophotometer at $505 \mathrm{~nm}$. The inhibition of the produced chromogen is proportional to the activity of the SOD present in the

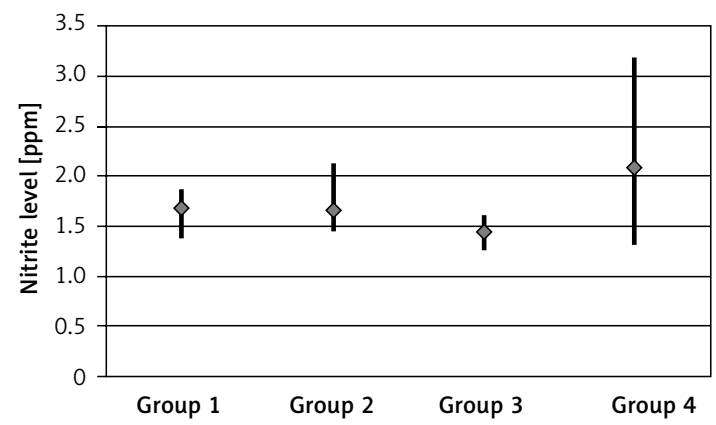

Figure 1. Nitrite levels by groups (group 1: control group, group 2: EPO group, group 3: EPO + RT group, group 4: RT group) sample. 50\% inhibition is defined as 1 unit of SOD (specific activity as units per milligram of protein).

The GSHPX activity was determined using the Ransel kit according to Paglia and Valentine [17]. GSHPX catalyzes the oxidation of glutathione by cumene hydroperoxide. In the presence of glutathione reductase and NADPH, the oxidized glutathione was immediately converted to the reduced form with concomitant oxidation of NADPH to NADP+. The decrease in absorbance was monitored with a spectrophotometer at $340 \mathrm{~nm}$. One GSHPX unit is defined as $1 \mu \mathrm{mol}$ of NADPH consumed per minute, and specific activity is reported as units per milligram of protein.

\section{Statistical analysis}

The results were expressed as means \pm standard deviation (SD) for the nitrite and MDA levels and SOD and GSHPX activities. Student's $t$ test was used for comparison. Statistical analysis was performed using SPSS (version 17.0), and $p<0.05$ was considered as significant.

\section{Results}

The nitrite levels were $1.69 \pm 0.14$ ppm (range: 1.4-1.87) for the control group, $1.66 \pm 0.23 \mathrm{ppm}$ (range: 1.47-2.13) for the EPO group, $1.45 \pm 0.12$ ppm (range: 1.27-1.60) for the EPO + RT group and $2.10 \pm 0.62$ ppm (range: $1.33-3.20$ ) for the RT group, and the difference was statistically significant $(p<0.05)$. There was a significant difference in nitrite levels among the control group and RT and EPO + RT groups; the nitrite level was higher in the RT group and lower in the EPO + RT group $(p<0.05)$ (Figure 1).

The MDA levels were $21.95 \pm 2.54 \mathrm{nmol} / \mathrm{ml}$ (range: 17.16-26.62) for the control group, 25.07 $\pm 3.89 \mathrm{nmol} / \mathrm{ml}$ (range: $18.26-33.22$ ) for the EPO group, $25.49 \pm 1.90 \mathrm{nmol} / \mathrm{ml}$ (range: $22.60-27.78$ ) for the EPO + RT group and $26.02 \pm 2.16 \mathrm{nmol} / \mathrm{ml}$ (range: 23.19-29.27) for the RT group, and the difference was statistically significant $(p<0.05)$. There was a significant difference in MDA levels between the control group and the RT group; the MDA level was higher in the RT group $(p<0.05)$ and lower in the EPO + RT group (Figure 2).

The SOD activity was $1.73 \pm 0.13 \mathrm{U} / \mathrm{mg}$ (range: 1.54-1.94) for the control group, $1.95 \pm 0.15 \mathrm{U} / \mathrm{mg}$ (range: 1.72-2.14) for the EPO group, $2.62 \pm 0.49$ $\mathrm{U} / \mathrm{mg}$ (range: 1.80-3.15) for the EPO + RT group and $1.90 \pm 0.17 \mathrm{U} / \mathrm{mg}$ (range: 1.62-2.14) for the RT group, and the difference was statistically significant $(p<0.05)$. There was a significant difference in SOD activity between the RT and EPO + RT groups; the SOD activity was higher in the EPO + RT group ( $p<0.05)$ (Figure 3 ).

The GSHPX activity was $1.67 \pm 0.16 \mathrm{U} / \mathrm{mg}$ (range: 1.33-1.86) for the control group, 1.54 


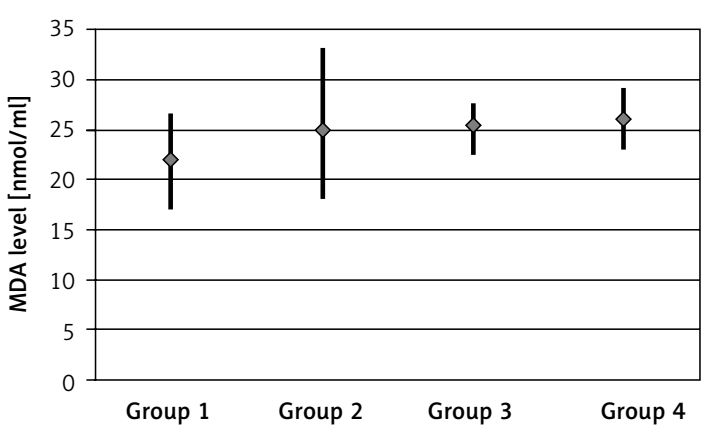

Figure 2. MDA levels by groups (group 1: control group, group 2: EPO group, group 3: EPO + RT group, group 4: RT group)

$\pm 0.18 \mathrm{U} / \mathrm{mg}$ (range: $1.12-1.75)$ for the EPO group, $1.75 \pm 0.25 \mathrm{U} / \mathrm{mg}$ (range: $1.37-1.99)$ for the EPO + RT group and $1.71 \pm 0.29 \mathrm{U} / \mathrm{mg}$ (range: $1.35-2.13$ ) for the RT group, and the difference was statistically significant $(p<0.05)$. There was increased activity in the EPO + RT group (Figure 4).

The relevant biochemical results are summarized in Table I.

\section{Discussion}

Radiation therapy is a major treatment modality that is received by about $60 \%$ of all cancer patients either for cure or palliation. The effectiveness of RT is limited by the potential for normal tissue injury. Radiation injury of the CNS has devastating clinical consequences. The associated pathologies are thought to be a consequence of complex interplay among many different processes including vascular endothelial cell loss, excessive generation of ROS and inflammatory mediators [18]. It is now recognized that clonogenic cell death is not the only mode of cell death in the CNS after RT $[19,20]$. Certain glial, neuronal and endothelial cells in the CNS also undergo apoptosis soon after RT. Furthermore, there is a component of secondary injury and cell death that is mediated by microenvironmental alterations such as hypoxia/ischemia and inflammation [1]. Similar to other CNS injury models associated

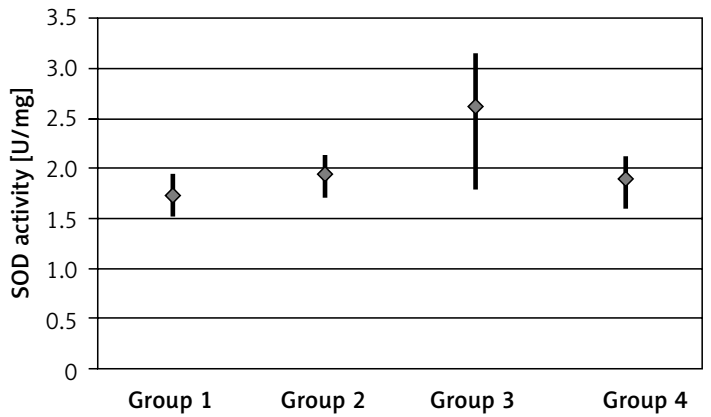

Figure 3. SOD activity by groups (group 1: control group, group 2: EPO group, group 3: EPO + RT group, group 4: RT group)

with an increase in ROS and a state of oxidative stress, the same was observed in the irradiated CNS. Similar with the literature in our study, the nitrite and MDA levels were increased in the RT group as an indicator of lipid peroxidation and presence of radiation-induced oxidative damage. Studies comparing MDA levels in rat brains after irradiation or sham irradiation showed that MDA levels for the rats that underwent irradiation were significantly higher than for the rats that underwent sham irradiation or sham surgery [21, 22]. High levels of MDA are indicative of oxidative stress [23].

Strategies aimed at blocking the effector molecules or otherwise reducing oxidative stress are attractive for preventing or mitigating radiation-in-

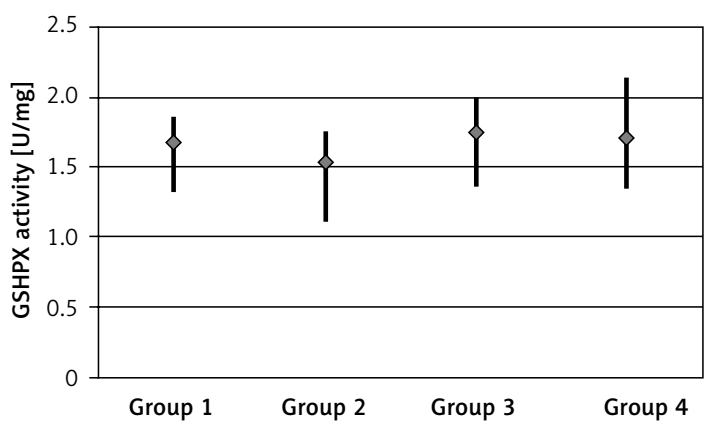

Figure 4. GSHPX activity by groups (group 1: control group, group 2: EPO group, group 3: EPO + RT group, group 4: RT group)

Table I. Nitrite and MDA levels and SOD and GSHPX activities by groups

\begin{tabular}{|lcccc|}
\hline Parameter & \multicolumn{3}{c|}{ Groups } \\
\cline { 2 - 5 } & Control & EPO & $\begin{array}{c}\text { EPO + radiation } \\
\text { therapy }\end{array}$ & Radiation therapy \\
\hline Nitrite $[\mathrm{ppm}]$ & $1.69 \pm 0.14$ & $1.66 \pm 0.23$ & $1.45 \pm 0.12$ & $2.10 \pm 0.62$ \\
& $(1.40-1.87)$ & $(1.47-2.13)$ & $(1.27-1.60)$ & $(1.33-3.20)$ \\
\hline MDA $[\mathrm{nmol} / \mathrm{ml}]$ & $21.95 \pm 2.54$ & $25.07 \pm 3.89$ & $25.49 \pm 1.90$ & $26.02 \pm 2.16$ \\
& $(17.16-26.62)$ & $(18.26-33.22)$ & $(22.60-27.78)$ & $(23.19-29.27)$ \\
\hline SOD $[\mathrm{U} / \mathrm{mg}]$ & $1.73 \pm 0.13$ & $1.95 \pm 0.15$ & $2.62 \pm 0.49$ & $1.90 \pm 0.17$ \\
& $(1.54-1.94)$ & $(1.72-2.14)$ & $(1.80-3.15)$ & $(1.62-2.14)$ \\
\hline GSHPX $[\mathrm{U} / \mathrm{mg}]$ & $1.67 \pm 0.16$ & $1.54 \pm 0.18$ & $1.75 \pm 0.25$ & $1.71 \pm 0.29$ \\
& $(1.33-1.86)$ & $(1.12-1.75)$ & $(1.37-1.99)$ & $(1.35-2.13)$ \\
\hline
\end{tabular}


duced CNS toxicity. One approach to impeding the actions of reactive oxidants is to administer drugs that induce endogenous antioxidant biochemical processes.

Human recombinant EPO has been used clinically for more than two decades to treat anemia associated with conditions such as chronic renal disease, in antiviral HIV therapy, and in cancer patients on chemotherapy. In an attempt to augment oxygen delivery as a response to hypoxia, EPO exerts its action through the up-regulation of specific receptors that have been shown to be abundant not only in the bone marrow, but also in a variety of tissues including the brain. Besides the expression the EPO receptors in the brain, autocrine production of EPO has been reported in the astrocytes as well as the oligodendrocytes, the glial cells and the endothelial cells [24].

In studies concerning the clinical use of EPO to correct anemia in patients with chronic renal diseases and cancer, an emphasis has been placed on the improvement in the quality of life as well as the improvement in the cognitive function [12, $25,26]$. Consequently, the effects of EPO on the quality of life and the cognitive function were evaluated in a placebo-controlled randomized pilot study by O'Shaughnessy et al. on patients with breast cancer undergoing chemotherapy, and EPO was found to attenuate the cognitive impairment associated with chemotherapy [27]. These clinical observations have indicated a direct potential neuroprotective role of EPO, and numerous studies are in favor of a neuroprotective role of EPO.

There is now an emerging body of data on the neuroprotective effect of EPO against a wide variety of CNS insults [28]. Local production of endogenous EPO in the brain in a hypoxia-dependent manner further suggests that EPO may act in a paracrine or autocrine manner to provide neuroprotection. Analogous to its activity in erythroid progenitor cells, EPO can stimulate the survival and proliferation of neural progenitor cells. Erythropoietin neuroprotection in animal models of brain ischemia/trauma raise the possibility for EPO therapy in brain injury/disease [29].

Erythropoietin use in stroke patients is appealing since it is well studied. Intravenous high-dose EPO in a total of 53 stroke patients was well tolerated and associated with an improvement in clinical outcome without any signs of elevated hematocrit levels [30]. With such encouraging early results, EPO investigations are being expanded to include Parkinson's disease, HIV-mediated neuropathy, diabetic neuropathy, macular degeneration, retinitis pigmentosa, glaucoma, demyelinating injury, schizophrenia, and other types of neurodegenerative diseases [31, 32].
Therefore, a potential neuroprotective role has been advocated for EPO in the setting of a variety of conditions affecting the central nervous system.

The suspected neuroprotective effects of EPO include a decrease in ROS generation, an increase in the expression of antioxidant enzymes, augmentation of the release of neurotransmitters, increased pro-angiogenic activity and the induction of neuroglobin (an intracerebral $\mathrm{O}_{2}$ transporter), hence antagonizing the deleterious action of glutamate, the inhibition of apoptosis [12]. This vast array of protective properties, most likely related to the prominent role of EPO during brain development, may explain the potent neuroprotective action of EPO in various different animal models. These, mostly rodent, models range from hypoxia/ischemia to traumatic injury of the brain or spinal cord, subarachnoid hemorrhage, multiple sclerosis, Parkinson's disease, epilepsy, retina degeneration, neuropathies including diabetes, radiation-induced brain damage, and cerebral malaria, to mention just the most obvious examples [33].

Erythropoietin may also decrease NO-mediated injury, but it does not affect NO levels directly. The mechanisms involve reduced lipid peroxidation and increased glutathione peroxidase [34, 35]. In our study, the nitrite and MDA levels were lower in the EPO group than in the RT group. The SOD and GSHPX activity was higher in the EPO-treated group.

Despite a large molecular size and a highly glycosylated and negatively charged structure, EPO has been shown to cross the blood-brain barrier in a dose-dependent manner [13]. After a single high-dose intravenous injection, EPO is detectable in the brain within hours, reaching a peak concentration at 3-4 hours in the brain or cerebrospinal fluid in humans and other animals [24].

Based on experimental studies, EPO is neuroprotective starting at a dose of $5000 \mathrm{IU} / \mathrm{kg}$ [13]. Thus, following a high EPO dose (5000 U/kg i.v.) there was a peak in the CSF $4 \mathrm{~h}$ after injection, and a significant correlation was observed between blood and CSF EPO concentrations [12]. Thus, we used this dose in our study.

In conclusion, this study supports the probable neuroprotective effects of erythropoietin against oxidant injury following brain irradiation in a rat model, presumably through decreasing free radical production and increasing expression of antioxidant enzymes. Nevertheless, the use of EPO as a neuroprotectant for radiation-induced oxidant injury warrants further evaluation regarding both effectiveness and safety.

\section{Acknowledgments}

We thank Neslihan Yucel, M.D. for her invaluable assistance. 


\section{Conflict of interest}

The authors declare no conflict of interest.

\section{References}

1. Wong CS, Van der Kogel AJ. Mechanisms of radiation injury to the central nervous system: implications for neuroprotection. Mol Interv 2004; 4: 273-84.

2. Erkal HS, Batcioglu K, Serin M, Uyumlu B, Yucel N. The evaluation of the oxidant injury as a function of time following brain irradiation in a rat model. Neurochem Res 2006; 31: 1271-7.

3. Robbins MEC, Zhao W. Chronic oxidative stress and radiation-induced late normal tissue injury: a review. Int J Radiat Biol 2004; 80: 251-9.

4. Kim JH, Brown SL, Jenrow KA, Ryu S. Mechanisms of radiation-induced brain toxicity and implications for future clinical trials. J Neurooncol 2008; 87: 279-86.

5. Folkes LK, O'Neill P. Modification of DNA damage mechanisms by nitric oxide during ionizing radiation. Free Radic Biol Med 2013; 25: 578-85.

6. Halliwell B, Gutteridge JM. Oxygen toxicity, oxygen radicals, transition metals and disease. Biochem J 1984; 219: 1-14.

7. Riley PA. Free radicals in biology: oxidative stress and the effects of ionizing radiation. Int J Radiat Biol 1994; 65: 27-33.

8. Reis GS, Augusto VS, Silveira AP, et al. Oxidative-stress biomarkers in patients with pulmonary hypertension. Pulm Circ 2013; 3: 856-61.

9. Uyumlu AB, Erkal HS, Batcioğlu K, Serin M, Yucel N. Evaluation of oxidant injury induced by irradiation in brain tissues of rats of different ages. Balkan Med J 2009; 26: 237-43.

10. Nadam J, Navarro F, Sanchez P, et al. Neuroprotective effects of erythropoietin in the rat hippocampus after pilocarpine-induced status epilepticus. Neurobiol Dis 2007; 25: 412-26.

11. Ranchon Cole I, Bonhomme B, Doly M. Pre-treatment of adult rats with high doses of erythropoietin induces caspase- 9 but prevents light-induced retinal injury. Exp Eye Res 2007; 85: 782-9.

12. Milano M, Collomp R. Erythropoietin and neuroprotection: a therapeutic perspective. J Oncol Pharm Pract 2005; 11: 145-9.

13. Statler PA, McPherson RJ, Bauer LA, Kellert BA, Juul SE. Pharmacokinetics of high-dose recombinant erythropoietin in plasma and brain of neonatal rats. Pediatr Res 2007; 61: 671-75.

14. Stahr, HM. Analytical Toxicology Methods Manual. Iowa State University Press. Ames, Iowa, USA, 1977; 68-71.

15. Mihara M, Uchiyama $M$. Determination of malonaldehyde precursor in tissues by thiobarbituric acid test. Anal Biochem 1978; 86: 271-8.

16. Suttle NF. Copper deficiency in ruminants; recent developments. Vet Rec 1986; 119: 519-22.

17. Paglia DE, Valentine WN. Studies on the quantitative and qualitative characterization of erythrocyte glutathione peroxidase. J Lab Clin Med 1967; 70: 158-69.

18. Tofilon PJ, Fike JR. The radioresponse of the central nervous system: a dynamic process. Radiat Res 2000; 153 : 357-70.

19. Li YQ, Guo YP, Jay V, Stewart PA, Wong CS. Time course of radiation-induced apoptosis in the adult rat spinal cord. Radiother Oncol 1996; 39: 35-42.
20. Pena LA, Fuks Z, Kolesnick RN. Radiation-induced apoptosis of endothelial cells in the murine central nervous system: protection by fibroblast growth factor and sphingomyelinase deficiency. Cancer Res 2000; 60: 321-7.

21. Erol FS, Topsakal C, Ozveren MF, et al. Protective effects of melatonin and vitamin $E$ in brain damage due to gamma radiation: an experimental study. Neurosurg Rev 2004; 27: 65-9.

22. Peker S, Abacioglu U, Sun I, Yuksel M, Pamir MN. Irradiation after surgically induced brain injury in the rat: timing in relation to severity of radiation damage. J Neurooncol 2004; 70: 17-21.

23. Tomic S, Brkic S, Maric D, Mikic AN. Lipid and protein oxidation in female patients with chronic fatigue syndrome. Arch Med Sci 2012; 8: 886-91.

24. Kaneko N, Kako E, Sawamoto K. Enhancement of ventricular-subventricular zone-derived neurogenesis and oligodendrogenesis by erythropoietin and its derivatives. Front Cell Neurosci 2013; 7: 235.

25. Lundin AP, Delano BG, Quinn-Cefaro R. Perspectives on the improvement of quality of life with epoetin alfa therapy. Pharmacotherapy 1990; 10: 22S-6S.

26. Pawlicki M, Jassem J, Bösze P, et al. A multicenter study of recombinant human erythropoietin (epoetin alpha) in the management of anemia in cancer patients receiving chemotherapy. Anticancer Drugs 1997; 8: 949-57.

27. O'Shaughnessy JA, Vukelja SJ, Holmes FA, et al. Feasibility of quantifying the effects of epoetin alfa therapy on cognitive function in women with breast cancer undergoing adjuvant or neoadjuvant chemotherapy. Clin Breast Cancer 2005; 5: 439-46.

28. Goldman SA, Nedergaard M. Erythropoietin strikes a new cord. Nat Med 2002; 8: 785-7.

29. Noguchi CT, Asavaritikrai P, Teng R, Jia Y. Role of erythropoietin in the brain. Crit Rev Oncol Hematol 2007; 64: 159-71.

30. Ehrenreich H, Hasselblatt M, Dembowski C, et al. Erythropoietin therapy for acute stroke is both safe and beneficial. Mol Med 2002; 8: 495-505.

31. Becerra SP, Amaral J. Erythropoietin: an endogenous retinal survival factor. N Engl J Med 2002; 347: 1968-70.

32. Lipton SA. Erythropoietin for neurologic protection and diabetic neuropathy. N Engl J Med 2004; 350: 2516-17.

33. Ehrenreich H, Bartels C, Sargin D, Stawicki S, Krampe H. Recombinant human erythropoietin in the treatment of human brain disease: focus on cognition. J Ren Nutr 2008; 18: 146-53

34. Katavetin P, Tungsanga K, Eiam-Ong S, Nangaku M. Antioxidative effects of erythropoietin. Kidney Int Suppl 2007; 107: S10-15.

35. Genc S, Akhisaroglu M, Kuralay F, Genc K. Erythropoietin restores glutathione peroxidase activity in 1-methyl-4-phenyl-1,2,5,6-tetrahydropyridine-induced neurotoxicity in C57BL mice and stimulates murine astroglial glutathione peroxidase production in vitro. Neurosci Lett 2002; 321: 73-6. 